\title{
The Geodesic Carathéodory Number
}

\author{
Eduardo S. Lira ${ }^{1}$, Diane Castonguay ${ }^{1}$, Erika M. M. Coelho ${ }^{1}$, Hebert Coelho ${ }^{1}$ \\ ${ }^{1}$ Instituto de Informática - Universidade Federal de Goiás (UFG) \\ Alameda Palmeiras, Quadra D, Câmpus Samambaia - 74.690-900 - Goiânia - GO - Brazil \\ \{eduardolira, diane, erikamorais, hebert\}@inf.ufg.br
}

\begin{abstract}
From Carathéodory's theorem arises the definition of the Carathéodory number for graphs. This number is well-known for monophonic and Triangle-path convexities. It is limited for some classes of graphs on $P_{3}$ and geodesic convexities but is known to be unlimited only on $P_{3}$-convexity. In this paper, we prove that the Carathéodory number is unlimited on geodesic convexity.
\end{abstract}

Resumo. Do teorema de Carathéodory surge a definição do número de Carathéodory para grafos. Este número é bem conhecido nas convexidades monofônica e de caminho de triângulos. Ele é limitado para algumas classes de grafos nas convexidades $P_{3}$ e geodésica, mas apenas na convexidade $P_{3}$ sabe-se que ele é ilimitado. Neste artigo, nós provamos que o número de Carathéodory é ilimitado na convexidade geodésica.

\section{Introduction}

In 1911, Constantin Carathéodory published a theorem stating that every $u$ in the convex hull of a subset $S \subseteq R^{d}$, is also in the convex hull of a subset $F$ of $S$ of order at most $d+1$ [Carathéodory 1911]. The ideas provenient from this theorem, brought to light an invariant in graph theory, called Carathéodory number, widely studied since then. We will focus on the study of this number on geodesic convexity.

In this article, we used the following definition for the Carathéodory number of a graph. A subset $S$ of vertices is a Carathéodory set if there exists a vertex in the convex hull of $S$ which does not belong to the convex hull of the $S \backslash\{s\}$ for every $s \in S$. The Carathéodory number $c(G)$ of a graph $G$ is the maximum cardinality of a Carathéodory set [E. M. M. Coelho and Szwarcfiter 2013].

The Carathéodory number is well-known for some convexities. On monophonic convexity, $c(G)=1$ for complete graphs, and 2 for other graphs [Duchet 1988]. On Triangle-path convexity $c(G)=2$ [Changat and Mathew 1999]. On $P_{3}$-convexity, $c(G) \leq 3$ for multipartite tournaments [D.B. Parker and Wolf 2008], however, it is unlimited considering general graphs [E. M. M. Coelho and Szwarcfiter 2013]. Considering the geodesic convexity, [M.C. Dourado 2013] showed that it is NP-complete to determine whether $c(G)$ is at least a given $k$, even when restricted to bipartite graphs, and that $c(G)$ is at most 3 for split graphs.

This article brings a new result on this matter: the Carathéodory number is unlimited on geodesic convexity. 


\section{Preliminaries}

Let $G$ be a finite graph given by a set of vertices $V(G)$ and a set of edges $E(G)$. A set $\mathcal{C}$ of subsets of $V(G)$ is a convexity if $\emptyset, V(G) \in \mathcal{C}$ and $\mathcal{C}$ is closed under intersections. The elements of $\mathcal{C}$ are called convex sets. Given a set $S \subset V$, the convex hull of $S$ is the smallest convex set $H(S) \in \mathcal{C}$, such that $S \subseteq H(S)$.

Several convexities are defined by a set $\mathcal{P}$ of paths in a graph. In this scenario, a subset $\mathcal{C} \in V(G)$ is convex when for all $x, y \in \mathcal{C}$, if $p \in \mathcal{P}$ is a path between $x$ and $y$, then all vertices in $p$ are also in $\mathcal{C}$.

The geodesic convexity is obtained when $\mathcal{P}$ is the set of all geodesics, i.e., all the shortest paths. A chord of a path $p$ in a graph $G$ is any edge joining a pair of nonadjacent vertices of $P$. The monophonic convexity is defined using the set of all chordless paths, i.e., induced paths. The triangle-path convexity arise from the set of all paths in which every chord is a short chord, i.e., chords joining vertices at distance 2 apart. And the $P_{3}$-convexity, also known as two-path convexity, is obtained from the set of all paths of length 2.

The Carathéodory number of a graph $G$, written as $c(G)$, is the smallest integer $k$ such that for every subset $S$ of $V(G)$ and every element $s$ in $H(S)$, there is a subset $F$ of $S$ with $|F| \leq k$ and $u \in H(F)$.

A subset $S \subset V(G)$ is called Carathéodory set when $\partial H(S)=H(S) \backslash$ $\bigcup_{s \in S} H(S \backslash\{s\})$ is nonempty. We can also define the Carathéodory number as the maximum cardinality of a Carathéodory set [E. M. M. Coelho and Szwarcfiter 2013].

\section{Geodesic Carathéodory Number is Unlimited}

We now construct inductively a family of graphs $G_{i}$, with $i \geq 1$ that have an unlimited Carathédory number on geodesic convexity:

- $G_{1}$ is the graph with $V\left(G_{1}\right)=\left\{v_{1}\right\}$, and $E\left(G_{1}\right)=\emptyset$;

- $G_{2}$ is the graph with $V\left(G_{2}\right)=V\left(G_{1}\right) \cup\left\{v_{2}, v_{3}\right\}$, and $E\left(G_{2}\right)=E\left(G_{1}\right) \cup$ $\left\{v_{1} v_{3}, v_{2} v_{3}\right\}$

- $G_{3}$ is the graph with $V\left(G_{3}\right)=V\left(G_{2}\right) \cup\left\{v_{4}, v_{5}, v_{6}\right\}$, and $E\left(G_{3}\right)=E\left(G_{2}\right) \cup$ $\left\{v_{1} v_{4}, v_{2} v_{4}, v_{3} v_{4}, v_{3} v_{5}, v_{4} v_{6}, v_{5} v_{6}\right\}$

- $G_{i+1}$, for $i \geq 3$, is the graph with $V\left(G_{i+1}\right)=V\left(G_{i}\right) \cup\left\{v_{n-2}, v_{n-1}, v_{n}\right\}$, and $E\left(G_{i+1}\right)=E\left(G_{i}\right) \cup\left\{v_{n-4} v_{n-2}, v_{n-3} v_{n-2}, v_{n-2} v_{n-1}, v_{n-1} v_{n}, v_{4} v_{n}\right\}$ where $n=3 i$.

Theorem 1. Every $G_{i}$, constructed as above, has a Carathéodory set of cardinality $i$.

Proof. For $i=1, S_{1}=\left\{v_{1}\right\}$ is a Carathéodory set of $G_{1}$. For $i=2, S_{2}=\left\{v_{1}, v_{2}\right\}$ and $v_{3} \in \partial H\left(S_{2}\right)$. We will now show by induction that $S_{i}=\left\{v_{1}, v_{2}\right\} \cup\left\{v_{3 j} \mid 2 \leq\right.$ $j \leq i\}$ is a Carathéodory set of $G_{i}$, with cardinality $i$, for $i \geq 3$. For $i=3$, note that $v_{5} \in \partial H\left(S_{3}\right)$. For $i=4$, we have that $v_{7}, v_{8} \in \partial H\left(S_{4}\right)$ and for $i=5$, one can see that $\partial H\left(S_{5}\right)=\left\{v_{10}, v_{11}\right\}$. This proves that $S_{i}$ is a Carathéodory set of $G_{i}$, with cardinality $i$, for $1 \leq i \leq 5$.

Take $i \geq 5$ and assume (as inductive hypothesis) that the longest geodesic of $G_{i}$ is at most 4 , and $S_{i}$ is a Carathéodory set of cardinality $i$, with $\left\{v_{n-5}, v_{n-4}\right\}=\partial H\left(S_{i}\right)$, where $n=3 i$. 
Take $S_{i+1}=S_{i} \cup\left\{v_{n}\right\}$, and note that all vertices in $S_{i+1}$ are connected to $v_{4}$, and that any other vertex in $V\left(G_{i+1}\right)$ is connected to at least one vertex in $S_{i+1}$, by construction of $G_{i+1}$. Therefore, the longest geodesic of $G_{i+1}$ is at most 4 . There are three edges connecting the vertices in $V\left(G_{i}\right)$ to those new vertices added to build $G_{i+1}$, namely $v_{n-4} v_{n-2}, v_{n-3} v_{n-2}$, and $v_{4} v_{n}$. Choose any two vertices $x, y$ in $V\left(G_{i}\right)$, other than $v_{4}$ (note that the geodesic distance of any vertex to $v_{4}$ is at most 2). If you take a path between $x$ and $y$ that uses both edges $v_{n-4} v_{n-2}$ and $v_{n-3} v_{n-2}$, it is bigger then their geodesic in at least one unit, since $v_{n-4} v_{n-3} \in E\left(G_{i}\right)$. If you take a path between $x$ and $y$ that uses $v_{n-4} v_{n-2}$ or $v_{n-3} v_{n-2}$, and $v_{4} v_{n}$, this path is bigger than 4 . So, by inductive hypothesis, in $G_{i+1}$ no shorten path was introduced between two vertices of $G_{i}$, implying that $H\left(S_{i}\right)$ in $G_{i+1}$ is exactly $V\left(G_{i}\right)$.

All the geodesics between $v_{n}$ and another vertex in $S_{i+1}$ have size 2 , and between $v_{n}$ and another vertex not in $S_{i+1}$ have size at most 3 . Take any vertex in $V\left(G_{i}\right)$ that is not in $S_{i+1}$, other than $v_{n-4}$. If a path between such vertex and $v_{n}$ uses either edge $v_{n-4} v_{n-2}$ or $v_{n-3} v_{n-2}$, it is bigger than 3 , so it is not a geodesic. The geodesics between $v_{n-4}$ and $v_{n}$ are $v_{n-4} v_{n-2} v_{n-1} v_{n}$ and $v_{n-4} v_{n-3} v_{4} v_{n}$. This means the only minimum path between a vertex in $V\left(G_{i}\right)$ and $v_{n}$ that uses the vertices $v_{n-2}$ and $v_{n-1}$ is the one between $v_{n-4}$ and $v_{n}$. Without loss of generality, using the inductive hypothesis, we can conclude that $\partial H\left(S_{i+1}\right)=\left\{v_{n-2}, v_{n-1}\right\}$ (meaning $S_{i+1}$ is a Carathéodory set), and $\left|S_{i+1}\right|=i+1$, completing the proof.

Corollary 1. The Carathéodory number on geodesic convexity is unlimited.

Proof. By Thereom 1, for all integer $i \geq 1$, there is a Carathéodory set of cardinality $i$ in $G_{i}$. We conclude that $c\left(G_{i}\right) \geq i$ and thus yields the results.

\section{References}

Carathéodory, C. (1911). Uber den variabilitätsbereich der fourierschen konstanten von positiven harmonischen funktionen. In Rend. Circ. Mat. Palermo, pages 193 - 217.

Changat, M. and Mathew, J. (1999). On triangle path convexity in graphs. In Discrete Math, pages 91-95.

D.B. Parker, R. W. and Wolf, M. (2008). On two-path convexity in multipartite tournaments. In European J. Combin., pages 641-651.

Duchet, P. (1988). Convex sets in graphs ii. minimal path convexity. In J. Combin Theory, Ser. B, pages 307-316.

E. M. M. Coelho, M.C. Dourado, D. R. and Szwarcfiter, J. (2013). The carathéodory number of the $p_{3}$ convexity of chordal graphs. In The Seventh European Conference on Combinatorics, Graph Theory and Applications, pages 209-214.

M.C. Dourado, D. Rautenbach, e. a. (2013). On the carathéodory number of interval and graph convexities. In Theoretical Computer Science, pages 127-135. 